\title{
Telepsychiatry in South Africa - present and future
}

Sinclair Wynchank, MB ChB, MD, D Phil, F Inst P, C Eng Telemedicine Platform, Medical Research Council, Cape Town, and School of Child and Adolescent Health, University of Cape Town

Jill Fortuin, B OH, MSC

Telemedicine Platform, Medical Research Council, Cape Town

The recent progress in telepsychiatry results from advancing technology and videoconferencing as an effective alternative to classic face-to-face psychiatric interaction, in terms of both results and costeffectiveness. The wide range of applications of telepsychiatry is outlined - in particular, emergency, forensic, prison and adolescent psychiatry. Attitudes of patients and the providers of psychiatric services are discussed, and a short account is given of the place of tele-education in psychiatric teaching. The advantages of telepsychiatry are outlined and its limitations are discussed. Lastly, the limited experience and relevance of telepsychiatry in South Africa (and hence for other developing countries) are presented. The conclusion is that telepsychiatry is well established, its successes documented, and that it should be applied in planning future public health services in South Africa.

Telepsychiatry is one of the most longstanding telemedicine disciplines, having started over a century ago with the inception of the telephone; its modern form dates from about 1960. Telepsychiatry uses information and computer technology for diagnosis, therapy, follow-up, forensic purposes, pharmacotherapy management, psychiatric education, etc. Telecommunication links between a remote site and another to which a request/referral is made, allow data transfer (e.g. of videoconferencing images, history, reports and teaching material). Links range from telephone lines to rapid-transfer wireless links. Two or more persons at different locations (typically, a psychiatrist and a patient) most frequently use interactive videoconferencing for the provision of psychiatric expertise not otherwise available at the sender's (i.e. patient's) location. Enormous progress in telepsychiatry and all forms of telemedicine has been made recently, owing to rapidly decreasing costs of computing power, and larger data transfer capacity and storage, which allow telepsychiatry to provide unprecedented access to mental health services, and especially in rural areas.

\section{Videoconferencing $v$. face-to-face}

Psychiatry is so firmly associated with face-to-face interaction that telepsychiatry's successes are insufficiently appreciated. Many comparisons have been made between telepsychiatric videoconferencing and face-to-face interactions, with the former usually achieving similar, or better, results. Patients are equally or more satisfied with telepsychiatry, and generally there is better follow-up. ${ }^{2}$ Comparisons, when the same psychiatrist used both modalities, show similar success rates for cognitive behaviour therapy and psychotropic medication administration. Differences in compliance for both the medication plan and follow-up appointments have favoured telepsychiatry. Comparing video-conferencing, telephone consultations and conventional face-to-face methods, satisfactory assessment was possible with all three modes for depression, using the Montgomery-Asberg Depression Rating Scale. ${ }^{3}$ Telepsychiatry has also been successful in child psychiatry, depression, dementia, schizophrenia, suicide prevention, post-traumatic stress, panic disorders, substance abuse, eating disorders and smoking prevention. ${ }^{4}$

\section{Applications of telepsychiatry}

Telephonic interaction has a long history in telepsychiatry, and still remains important, as recent studies in the USA attest. In one report, $15 \%$ of such calls were 'urgent' and $1.5 \%$ were 'emergencies'. In the UK land many other countries, including South Africa) patients with mental illness are often cared for by primary health care $(\mathrm{PHC})$ providers 5 for whom a supplementary telepsychiatry service (even telephonic) can be most valuable for complex and acute situations. Additionally, telepsychiatric videoconferencing can be used for interacting with patients and also for administration, pharmacological management, education, training and research activities. "Depression has often been managed successfully via telepsychiatry, especially in elderly white men with substantial physical and behavioural health comorbidity.? Telepsychiatric treatment for comorbid depression with either a problematic cannabis or alcohol dependence has been shown to be equally or more effective (for outcomes after a year) than traditional interventions. ${ }^{8}$ Other conditions amenable to telepsychiatric management include sleep disorders and postpartum depression. 


\section{Forensic and prison psychiatry}

Many reports describe forensic and prison telepsychiatry. Investigations in the USA to establish competency to stand trial, using a standard test via videoconferencing, established it as 'a reliable method of assessment', satisfied with telepsychiatry and face-to-face interaction. Overall, telepsychiatry was considered fully acceptable, whether for civilian proceedings (such as commitment) or to determine fitness for a criminal trial. A legal appeal against a court decision that depended on telepsychiatry was rejected, for it was deemed that telepsychiatry was 'not violating due process'. 9 In Australia, videoconferencing provides timely expert advice to courts in remote areas, with assessments usually available within an hour; the resulting costs were $1 / 200$ to $1 / 400$ of conventional costs in obtaining expert opinions. ${ }^{10}$ These techniques apply equally to paediatric interactive court testimony. " Potential problems, if anticipated, can normally be overcome, e.g. unauthorised access to interview recordings, data transmission, or influence on the witness by persons in the same room but out of the camera's field.

Psychiatric morbidity in prisons is high; in one report from Norway, $25 \%$ of prisoners required psychiatric interventions, $20 \%$ needed psychotherapy, and $3 \%$ had psychotic disorders. ${ }^{12}$ For prisoners, telemental health 'is an efficient treatment modality', with prisoners having no significant difference in preference for either method. ${ }^{13}$ Prisoners also prefer telepsychiatry for sexual or safely issues; for other matters, on-site mental health staff, if they exist, are preferred. The range of problems successfully treated using telepsychiatry for prisoners is wide, and includes adjustment, substance, mood and anxiety disorders; behavioural dyscontrol; negative affect; interpersonal and institutional relations; self-preservation; self-reliance and procedural concerns. Adolescent prisoners expressed similar satisfaction with telepsychiatry, but some concerns about privacy. ${ }^{14}$ Many psychiatric problems (especially substance abuse and emotional and behavioural disorders) were satisfactorily managed for the latter. Some advantages in all cases are transport cost savings, security considerations, successfully supervised pharmacotherapy, and elimination of the shame of wearing handcuffs and other restraints in a public place.

\section{Emergency psychiatry}

Telepsychiatry can be effective in two types of emergency: unique clinical events and mass disasters. ${ }^{15}$ In isolated, remote and rural regions with limited resources, the risk of poor outcomes, such as murder and suicide, is higher. Emergency psychiatric services, using interactive videoconferencing, can assess and treat patients and also be preferable for face-to-face interaction with potentially dangerous patients. ${ }^{16}$ A Finnish emergency psychiatric service was considered 'comparable to face-to-face' and 'suitable for the assessment of psychiatric emergency patients and satisfactory for patients and staff alike', ${ }^{17}$ especially because there was no waiting for a consultation or travelling. After experiencing emergency telepsychiatry, most (90\%) patients indicated that they preferred it for subsequent consultations.

\section{Routine referrals}

Routine psychiatric referrals have also benefited from telepsychiatry, which has been described as 'a dependable method of assessment with a high degree of accuracy and substantial overall intermethod agreement, when compared with standard face-to-face interviews for new routine out-patient referrals'. Particularly for depression, it is comparable with face-to-face care in terms of treatment outcomes, patient adherence to treatment, patient satisfaction and health care costs. ${ }^{18}$

\section{Children and adolescents}

Telepsychiatry is as effective for juveniles as for adults, providing sufficient rapport and ability to aid diagnoses of psychopathology by way of evaluation, diagnosis and ongoing treatment for autism and oppositional defiant and anxiety disorders. ${ }^{19}$ 'Some parents reported that their child actually preferred telepsychiatry to conventional consultation. ${ }^{20}$ It was also considered a 'positive addition' to the mental health service in Scotland ${ }^{21}$ and to have the 'potential to be a useful treatment alternative' for child and adolescent psychiatry. ${ }^{22}$

\section{Education}

Telepsychiatric links can be used for training in clinical supervision; educating the patient, family and health care provider; and under- and postgraduate student instruction. Most importantly, one of the aims of any rural telepsychiatry service is 'to educate and upgrade the skills of primary care providers to allow them to manage a greater proportion of their patients with psychiatric illnesses, using best-practice principles, without the need for specialist psychiatrist support'. ${ }^{23}$ Various reports indicate that this has been achieved, especially in trainee supervision and continuing professional education. ${ }^{23,24} \mathrm{~A}$ review of USA university telepsychiatry education has highlighted Harvard's interactive case-based online network and flexible web-based curricula at the Universities of California (San Francisco) and Missouri. ${ }^{25} \mathrm{~A}$ telepsychiatric clinic has also been shown to be an appropriate setting for direct supervision of trainees. ${ }^{26}$ 
Palliative care for patients and families often involves psychiatry. The only African university courses in palliative medicine la diploma and MPhil) that depend principally on tele-education techniques are offered by the University of Cape Town, and attract students from 12 African countries (personal communication, E Gwyther). A joint project between the Medical Research Council and the Department of Psychology at the University of the Western Cape involved an audiovisual link between the university campus and a PHC nurse-directed clinic in Beaufort West. The link allowed assessment when agitated patients presented, especially over weekends; they would otherwise be taken to local police cells until a district surgeon visited the following Monday. The link was also used for teaching purposes on the campus, using clinic patients whose pathology was rarely encountered in the department. Since such links are bidirectional, they allow use of distance learning techniques to train two types of volunteer lay-counsellor: professional people (clergymen, librarians, nurses, teachers, social workers, etc.) and senior high-school students; each group is trained separately. The latter were particularly effective in counselling fellow pupils. Regrettably, this successful programme ended prematurely after the equipment was stolen.

\section{Limitations of telepsychiatry}

Problem areas include reluctance of practitioners to adopt new technology, how to maintain privacy and confidentiality, ensuring reimbursement, and establishing methods for and maintenance of appropriate standards of care. Such problems are not always easy to resolve, so the uptake of telepsychiatry has been slower than expected.

A complete course of psychotherapy may take longer when using telepsychiatry. ${ }^{27}$ Possible reasons for this are related to understanding successful psychotherapy, which establishes a rapport between patient and therapist, where trust, honesty and professional skills allow exchanges that benefit patients. Since videoconferencing is often unfamiliar to patients (even if faces and voices are not greatly distorted), it may take longer to establish the necessary rapport. Many patients may have difficulty in expressing feelings to a 'screen therapist'. Occasionally, technical problems may affect the frequency of sessions. Such factors can affect the rate of progress, and consequently it can take longer to complete treatment for new referrals managed by telepsychiatry. In the USA (especially in rural areas), the greatest limitation encountered so far in expanding telepsychiatry services is difficulty in ensuring reimbursement. ${ }^{28}$ Some patient-caregiver relationships may suffer with telepsychiatry - for example, in hospice and nursing home telecare, where the reduction of human contact can be detrimental, if telepsychiatry is substituted for faceto-face interaction. Other potential limitations of telepsychiatry may be encountered when promoting independence in place of dependence, maintaining privacy and confidentiality of patient data, obtaining informed consent and ensuring equity of access. However, these issues do not detract significantly from telepsychiatry's overall success.

\section{Advantages of telepsychiatry}

Psychiatry, more than most other forms of medical practice, must take account of cultural norms. The telepsychiatrist is usually an urban dweller; where the patient is rural, insufficient knowledge of rural culture may reduce the effectiveness of their interaction. There may be problems with non-verbal cues, especially 'eye contact, posture, facial expressions, body positioning, voice quality and tone, and hesitations, all of which can be important in assessing emotion'. Although this potential difficulty may cause a perception of lack of empathy, some feel that this is an advantage, since potentially distracting actions are out of view. ${ }^{5}$ Another possible advantage results from the distancing effects of videoconferencing, which can offer a sense of security and encourage honesty, particularly for schizophrenic and agarophobic patients. These effects may be because videoconferencing is held to be less threatening than personal contact, and such patients feel they can leave at any time, with little or no offence. They also tend to be less inhibited about problem discussion, since the interaction takes place on neutral ground, so allowing patients to feel they have more control over the situation. ${ }^{5}$ The greatest benefit of telepsychiatry is time and cost savings, since less-to-no travelling is required. It allows psychiatric services in regions that would otherwise have to do without them, and is a most effective substitute for traditional psychiatric interaction. The advantages more than compensate for the limitations.

A potential deficiency is in physical or neurological examination using telemedicine with a remote expert, because subtle signs may not be recognised. However, a remote interpreter of the examination, who is familiar with limitations of videoconferencing in this context, can often instruct the health care provider with the patient and assisting with the examination procedure, to pay particular attention to specific details, so improving the value of the examination. Establishing standardised comprehensive guidelines can greatly improve the efficacy of the telemedicine patient examination. ${ }^{15}$ In a study of remote specialist neurologists examining multiple sclerosis patients, it was found that agreement between telepsychiatry and face-to-face examination was 
satisfactory overall and similar to that reported for various neurological examiners directly assessing the same patient. However, such agreement was least consistent for brainstem and cerebellar functions. ${ }^{29}$

\section{Discussion}

Most patients of all ages, from children to geriatrics, give telepsychiatry high levels of satisfaction. Rural patients often have psychiatric care supplied by PHC providers, who may refer their patients, especially those with mood and anxiety disorders, for diagnosis and medication planning. Recent studies have shown that telepsychiatry improves (i) patient outcomes, (ii) their access to psychiatry in under-served areas, (iii) relevant knowledge and skills of PHC providers, and (iv) patient satisfaction from the former's care. ${ }^{30}$ Telepsychiatry's cost benefits have been confirmed by reports from various countries (e.g. Australia, the UK and Canada $^{31}$ ).

Use of telepsychiatry is about to increase in developing countries, in parallel with the boosting of other forms of telemedicine, as the same links can be used by many disciplines. Careful planning is essential to provide the most efficient application, as has been demonstrated in many current networks. In South Africa, experience with pilot telepsychiatry (and other telemedicine) links has confirmed the benefits of these techniques, as found elsewhere. South African public health service telepsychiatry is therefore likely to continue to expand.

\section{Conclusions}

Telepsychiatry is well established, with documented successes especially in remote rural populations and closed communities such as prisons, largely thanks to reduced travel costs. However, it is not widely appreciated that telepsychiatric and face-toface interactions are generally equivalent in both efficacy and participant satisfaction; when the latter differ, it is often to the advantage of telepsychiatry. These facts should be brought to the attention of the planners of health services in South Africa, especially for remote rural regions which in the past have been shortchanged in having an inferior public health service.

\section{Acknowledgements}

The authors are grateful to Mr Ashley Bess for administrative assistance and to Drs E Gwyther and D Wynchank for informative discussions.

\section{References}

1. Jarvis-Selinger S, Chan E, Payne R, Plohman K, Ho K. Clinical telehealth across the disciplines: lessons learned. Telemed J E Health 2008; 14(7): 720-725.

2. Urness D, Wass M, Gordon A, Tian E, Bulger T. Client acceptability and quality of life-telepsychiatry compared to in-person consultation. J Telemed Telecare 2006; 12(5): 251-254

3. Kobak KA, Williams JB, Jeglic E, Salvucci D, Sharp IR. Face-to-face versus remote administration of the Montgomery-Asberg Depression Rating Scale using videoconference and telephone. Depress Anxiety 2008; $25(11$ 1): 91 3-919.

4. Hailey D, Roine R, Ohinmaa A. The effectiveness of telemental health applications: a review. Can J Psychiatry 2008; 53(1 1): 769-778.

5. Norman S. The use of telemedicine in psychiatry. J Psychiatr Ment Health Nurs 2006; 13(6): $771-777$

6. McGinty KL, Saeed SA, Simmons SC, Yildirim Y. Telepsychiatry and e-mental health services: potential for improving access to mental health care. Psychiatr Q 2006; 77(4): 335-342

7. Fortney JC, Pyne JM, Edlund MJ, et al. A randomized trial of telemedicine-based collaborative care for depression. J Gen Intern Med 2007; 22(8): 1086-1093.

8. Kay-Lambkin FJ, Baker AL, Lewin TJ, Carr VJ. Computer-based psychological treatment for comorbid depression and problematic alcohol and/or cannabis use: randomized controlled trial of clinical efficacy. Addiction 2009; 104(3): 378-388.

9. Manguno-Mire GM, Thompson JW, Shore JH, et al. The use of telemedicine to evaluate competency to stand trial: a preliminary randomized controlled study. J Am Acad Psychiatry Law 2007; 35(4): 481-489.

10. Brett A, Blumberg L. Video-linked court liaison services: forging new frontiers in psychiatry in Western Australia. Australas Psychiatry 2006; 14(1): 53-56.

1 1. Miller TW, Burton DC, Hill K, et al. Telepsychiatry: critical dimensions for forensic services. J Am Acad Psychiatry Law 2005; 33(4): 539-546.

12. Kielsberg $E$, Hartvig $P$, Bowitz $H$, et al. Mental health consultations in a prison population: a descriptive study. BMC Psychiatry 2006; 6: 27.

13. Morgan RD, Patrick AR, Magaletta PR. Does the use of telemental health alter the treatment experience? Inmates' perceptions of telemental health versus face-to-face treatment modalities. J Consult Clin Psychol 2008; 76(1): 158-162.

14. Myers K, Valentine J, Morganthaler R, Melzer S. Telepsychiatry with incarcerated youth. J Adolesc Health 2006; 38(6): 643-648.

15. Yellowlees P, Burke MM, Marks SL, Hilty DM, Shore JH. Emergency telepsychiatry. J Telemed Telecare 2008; 14(6): 277-281.

16. Shore JH, Hilty DM, Yellowlees P. Emergency management guidelines for telepsychiatry. Gen Hosp Psychiatry 2007; 29(3): 199-206.

17. Sorvaniemi $M$, Ojanen $E$, Santamäki $O$. Telepsychiatry in emergency consultations: a follow-up study of sixty patients. Telemed J E Health 2005; 11 (4): 439-44 1

18. Singh SP, Arya D, Peters T. Accuracy of telepsychiatric assessment of new routine outpatient referrals. BMC Psychiatry 2007; 7: 55.

19. Hampton T. Researchers provide psychiatric care from afar. JAMA 2006; 295(1): 21-23

20. Sulbacher $S$, Vallin T, Waetzig EZ. Telepsychiatry improves paediatric behavioural health care in rural communities. J Telemed Telecare 2006; 12(6): 285-288

21. Mitchell SA, Maclaren AT, Morton M, Carachi R. Professional opinions of the use of telemedicine in child \& adolescent psychiatry. Scott Med J 2009; 54(3): 13-16.

22. Paing WW, Weller RA, Welsh $B$, et al. Telemedicine in children and adolescents Curr Psychiatry Rep 2009; 11 (2): $114-119$

23. Hilty DM, Yellowlees PM, Nesbitt TS. Evolution of telepsychiatry to rural sites: changes over time in types of referral and in primary care providers' knowledge, skills and satisfaction. Gen Hosp Psychiatry 2006; 28(5): 367-373.

24. Hilty DM, Nesbitt TS, Kuenneth CA, Cruz GM, Hales RE. Rural versus suburban primary care needs, utilization, and satisfaction with telepsychiatric consultation. J Rural Health 2007; 23(2): 163-165.

25. Hilty DM, Alverson DC, Alpert JE, et al. Virtual reality, telemedicine, web and data processing innovations in medical and psychiatric education and clinical care. Acad Psychiatry 2006; 30(6): 528-533.

26. Szeftel R, Hakak R, Meyer $S$, et al. Training psychiatric residents and fellows in a telepsychiatry clinic: a supervision model. Acad Psychiatry 2008; 32(5): 393-399.

27. Mclaren P, Ahlbom J, Riley A, Mohammedali A, Denis M. The North Lewisham Telepsychiatry project: beyond the pilot phase. J Telemed Telecare 2002; 8(suppl 2) 98-100

28. Hilty DM, Cobb HC, Neufeld JD, Bourgeois JA, Yellowlees PM. Telepsychiatry reduces geographic physician disparity in rural settings, but is it financially feasible because of reimbursement? Psychiatr Clin North Am 2008; 31 (1): 85-94.

29. Kane RL, Bever CT, Ehrmantraut M, et al. Teleneurology in patients with multiple sclerosis: EDSS ratings derived remotely and from hands-on examination. J Telemed Telecare 2008; 14(4): 190-194.

30. Harley J. Economic evaluation of a tertiary telepsychiatry service to an island. $J$ Telemed Telecare 2006; 12(7): 354-357

31. Persaud DD, Jreige $S$, Skedgel $C$, et al. An incremental cost analysis of telehealth in Nova Scotia from a societal perspective. J Telemed Telecare 2005; 11 (2): 77-84 